\title{
The value of intraoperative ultrasonography during the resection of relapsed irradiated malignant gliomas in the brain
}

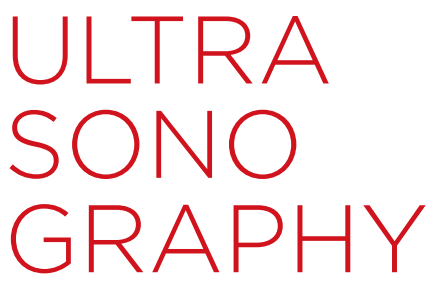

\author{
Kay Mursch', Martin Scholz², Wolfgang Brück², Julianne Behnke-Mursch ${ }^{1}$ \\ ${ }^{1}$ Department of Neurosurgery, Zentralklinik, Bad Berka; ${ }^{2}$ Department of Neurosurgery, \\ Klinikum Duisburg, Duisburg; ${ }^{3}$ Department of Neuropathology, Georg August Universität, \\ Göttingen, Germany
}

\section{ORIGINAL ARTICLE}

https://doi.org/10.14366/usg. 16015 pISSN: 2288-5919 - eISSN: 2288-5943 Ultrasonography 2017;36:60-65

Purpose: The aim of this study was to investigate whether intraoperative ultrasonography (IOUS) helped the surgeon navigate towards the tumor as seen in preoperative magnetic resonance imaging and whether IOUS was able to distinguish between tumor margins and the surrounding tissue.

Methods: Twenty-five patients suffering from high-grade gliomas who were previously treated by surgery and radiotherapy were included. Intraoperatively, two histopathologic samples were obtained a sample of unequivocal tumor tissue (according to anatomical landmarks and the surgeon's visual and tactile impressions) and a small tissue sample obtained using a navigated needle when the surgeon decided to stop the resection. This specimen was considered to be a boundary specimen, where no tumor tissue was apparent. The decision to take the second sample was not influenced by IOUS. The effect of IOUS was analyzed semi-quantitatively.

Results: All 25 samples of unequivocal tumor tissue were histopathologically classified as tumor tissue and were hyperechoic on IOUS. Of the boundary specimens, eight were hypoechoic. Only one harbored tumor tissue $(P=0.150)$. Seventeen boundaries were moderately hyperechoic, and these samples contained all possible histological results (i.e., tumor, infiltration, or no tumor).

Conclusion: During surgery performed on relapsed, irradiated, high-grade gliomas, IOUS provided a reliable method of navigating towards the core of the tumor. At borders, it did not reliably distinguish between remnants or tumor-free tissue, but hypoechoic areas seldom contained tumor tissue.

Keywords: Glioblastoma; Glioma; Interventional ultrasonography; Neoplasms, residual; Neurosurgical procedure

\section{Introduction}

Intraoperative ultrasonography (IOUS) has been used a tool in cranial neurosurgery for several years [1] but is not as popular as in other surgical disciplines due to certain limitations. In cranial neurosurgery, most of the region of interest is covered by bone, and only small image sectors are
Received: March 13, 2016

Revised: August 7, 2016

Accepted: August 8, 2016

Correspondence to:

Kay Mursch, MD, Department of Neurosurgery, Zentralklinik, RobertKoch-Allee 9, D-99438 Bad Berka, Germany

Tel. +49-36458-541313

Fax. +49-36458-53512

E-mail: kay.mursch@zentralklinik.de

This is an Open Access article distributed under the terms of the Creative Commons Attribution NonCommercial License (http://creativecommons.org/ licenses/by-nc/3.0/) which permits unrestricted noncommercial use, distribution, and reproduction in any medium, provided the original work is properly cited.

Copyright @ 2017 Korean Society of Ultrasound in Medicine (KSUM)

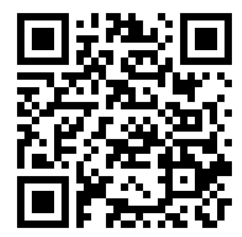

How to cite this article:

Mursch K, Scholz M, Brück W, Behnke-Mursch J. The value of intraoperative ultrasonography during the resection of relapsed irradiated malignant gliomas in the brain. Ultrasonography. 2017 Jan;36(1):60-65. 
accessible to the ultrasound beam. Thus, some advantages of interventional ultrasonography, such as hand-eye-coordination during movement of the probe, are not as valuable as in other organs. In addition, the brain is considered to be more sensitive to pressure than other tissue, which has been considered to be another limitation. The head position does not always allow the resection cavity to be filled completely with fluid, which may result in object artifacts. During recent years, advances in image data processing have led to improvements in image quality. IOUS can now be used to navigate towards deep brain lesions, to define the extent of resection, and to visualize tumor remnants to a certain degree [25]. Metastases, cavernous hemangiomas, and other well-delineated lesions are displayed with defined margins. However, the boundaries of infiltrating gliomas with the surrounding tissue cannot be distinguished properly $[3,6,7]$. Moreover, edema impairs the contrast between tumor and the surrounding tissue. It has been reported that IOUS images obtained within irradiated cerebral tissue may be very difficult to interpret and that the boundaries between relapsed glioma and tissue are poorly defined $[3,8]$.

In contrast to normal, hypoechoic brain tissue, irradiated brain tissue is slightly hyperechoic and somewhat "foggy." This study focuses specifically on the use of IOUS in treating malignant gliomas that had been previously been treated by radiation and operation. The aims of this study were to investigate whether IOUS helped the surgeon navigate towards the tumor as seen in preoperative magnetic resonance imaging (MRI) and whether IOUS was able to distinguish between a tumor margin and the surrounding tissue in these tumors.

\section{Materials and Methods}

Twenty-five patients ( 14 male, 11 female; aged between 42 to 74 years; median age, 62 years) suffering from high-grade gliomas (22 glioblastomas, 1 gliosarcoma, 1 anaplastic oligodendroglioma, and 1 anaplastic oligoastrocytoma), who had been previously treated with a single tumor resection (only 1 patient was operated on for the third time in the operation analyzed in this study), standard radiotherapy (usually with a dose of 59 to $60 \mathrm{~Gy}$ ) and by chemotherapy with temozolomide, were included in the study. The study was approved by our Institutional Review Board, and the requirement for informed consent was waived.

Local tumor relapse was diagnosed using contrast-enhanced MRI. The operations were performed using a microsurgical technique with the goal of gross tumor resection by a neurosurgeon with experience in IOUS. IOUS was used after craniotomy throughout the entire operation until the dura mater was closed. The ultrasound system was a Flex Focus 800 (BK, Herlev, Denmark). The problem of defining the biopsy region within the sonographic image was solved by using the integrated biopsy function within the ultrasound system, which is used for guided needle biopsy [9] but can be used to mark the region of biopsy.

Intraoperatively, two samples were obtained. Sample 1 was defined as unequivocal tumor tissue, according to the experienced surgeon's visual and tactile impressions. IOUS was used to identify intraoperative landmarks such as the first tumor approach, cysts, bone, and ventricles. Sample 2 was obtained when the surgeon was not sure about the tissue characteristics (i.e., whether it was tumor or normal tissue) and decided to stop the resection. This was considered a boundary sample, with no apparent tumor tissue. During this stage of the operation, IOUS was not used as a basis for surgical decisions, but only to mark the region of the biopsy and to characterize its echogenicity.

Video documentation was used to record the resection. When analyzing the images, we distinguished between three different types of tissue echogenicity: hypoechoic, moderately hyperechoic, and hyperechoic. For hypoechoic lesions similar to healthy brain tissue, a slight difference is present between white and gray matter on IOUS, but this was not noticeable to a problematic extent in our $5-7.5-\mathrm{MHz}$ images. For moderately hyperechoic lesions showing elevated echogenicity compared to white and gray brain tissue, the differential diagnosis was edema, radiated tissue, or tumor. For hyperechoic lesions with somewhat clear margins, the differential diagnosis was necrosis or tumor. The chi-square test was used to evaluate the statistical relationships between IOUS features and the pathologic diagnosis at the tumor boundary. P-values $<0.05$ were considered to indicate statistical significance.

\section{Results}

All 25 of the samples obtained from unequivocal tumor tissue were indeed classified as malignant glioma in the histopathological examination. All samples were resected from tissue that was hyperechoic on IOUS (Figs. 1, 2). The results for the second specimen are shown in Table 1. When the surgeon decided to stop the resection

Table 1. Results of the examination of the tumor borders (sample 2)

\begin{tabular}{lccc}
\hline \multirow{2}{*}{ Ultrasound image } & \multicolumn{3}{c}{ Result of histopathological examination } \\
\cline { 2 - 4 } & $\begin{array}{c}\text { Tumor } \\
(\mathrm{n}=8)\end{array}$ & $\begin{array}{c}\text { Infiltration } \\
(\mathrm{n}=9)\end{array}$ & $\begin{array}{c}\text { No tumor } \\
(\mathrm{n}=8)\end{array}$ \\
\hline Hypoechoic $(\mathrm{n}=8)$ & 1 & 4 & 3 \\
Moderately hyperechoic $(\mathrm{n}=17)$ & 7 & 5 & 5 \\
\hline
\end{tabular}

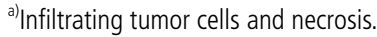




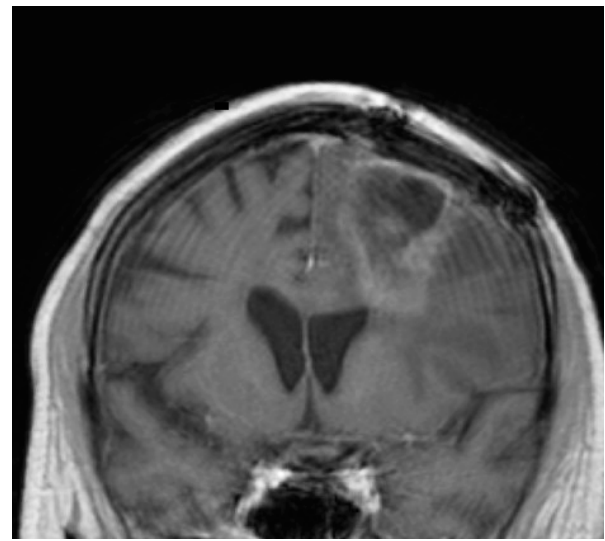

A

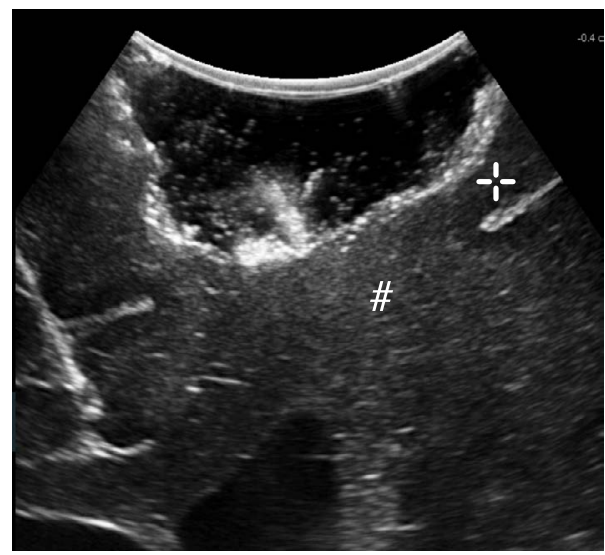

C

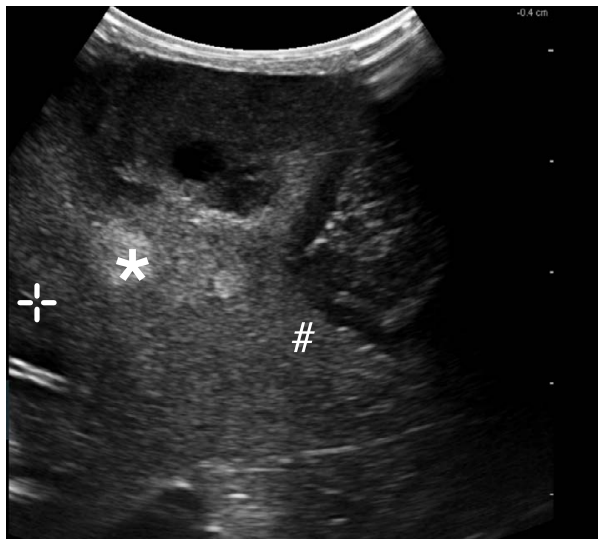

B

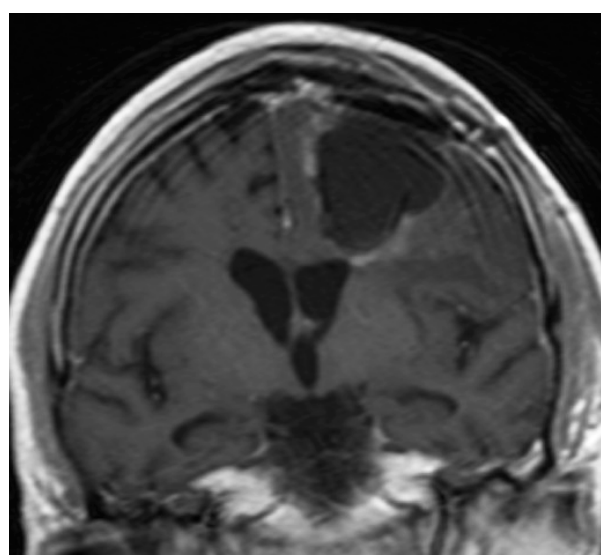

D
Tumor bulk

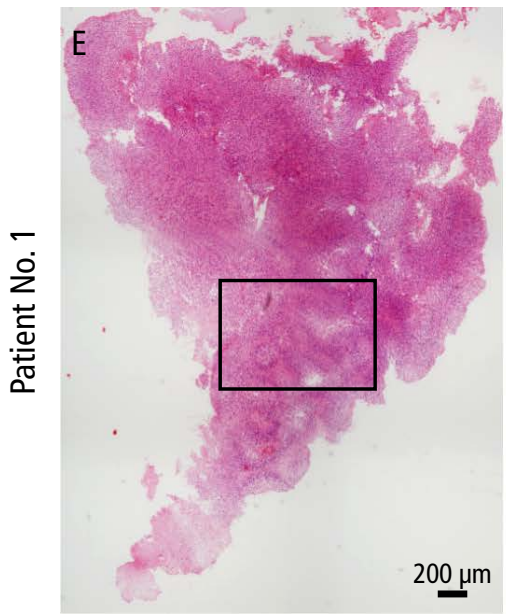

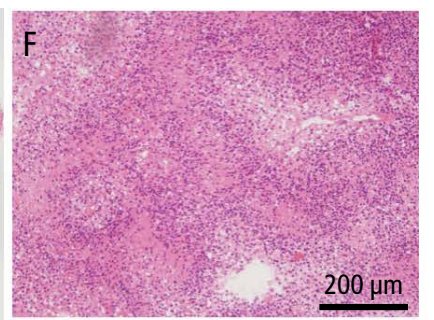

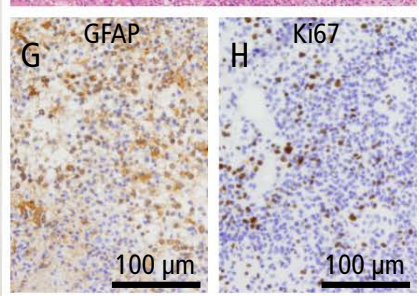

Tumor border

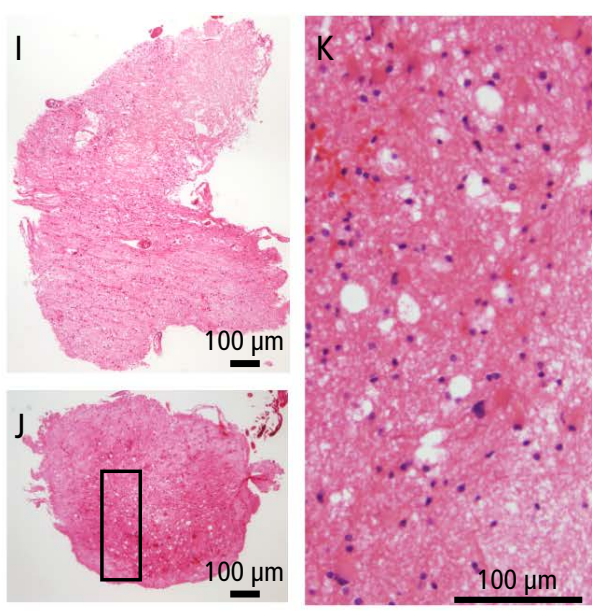

Fig. 1. A 60-year-old man with a right frontal glioblastoma, undergoing his second operation 16 months after the first operation.

A. Coronal magnetic resonance imaging (MRI) (T1, contrast-enhanced) defines the extent of tumor recurrence. B. Intraoperative ultrasonography (IOUS) before tumor resection (coronal image) reveals hypoechoic (+) as well as intermediate hypoechoic (\#) and hyperechoic tissue $(*)$. C. Coronal IOUS shows the extent of resection; at the border, a hypoechoic gyrus can be identified (+). The region where sample 2 was taken (indistinguishable to the surgeon's eye between border or normal tissue) is marked (\#). D. The postoperative coronal MRI (T1, contrast-enhanced) taken at the day after resection shows only linear contrast media enhancement. E-K. Histopathological examination demonstrated that the hyperechoic lesion contained cell-rich solid tumor (tumor bulk) (E-H), whereas in the intermediate tissue, infiltrating tumor cells and necrosis were found (tumor border) (I-K). 


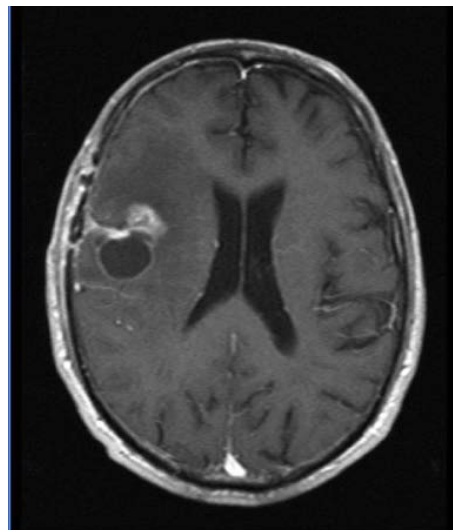

A
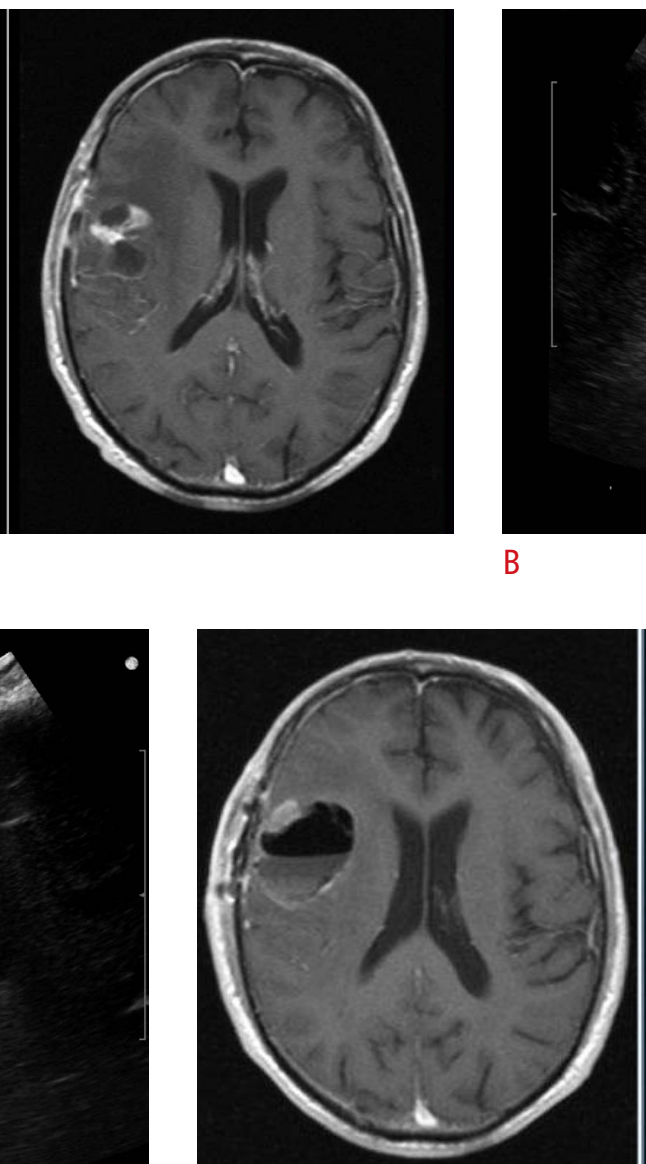

D

B
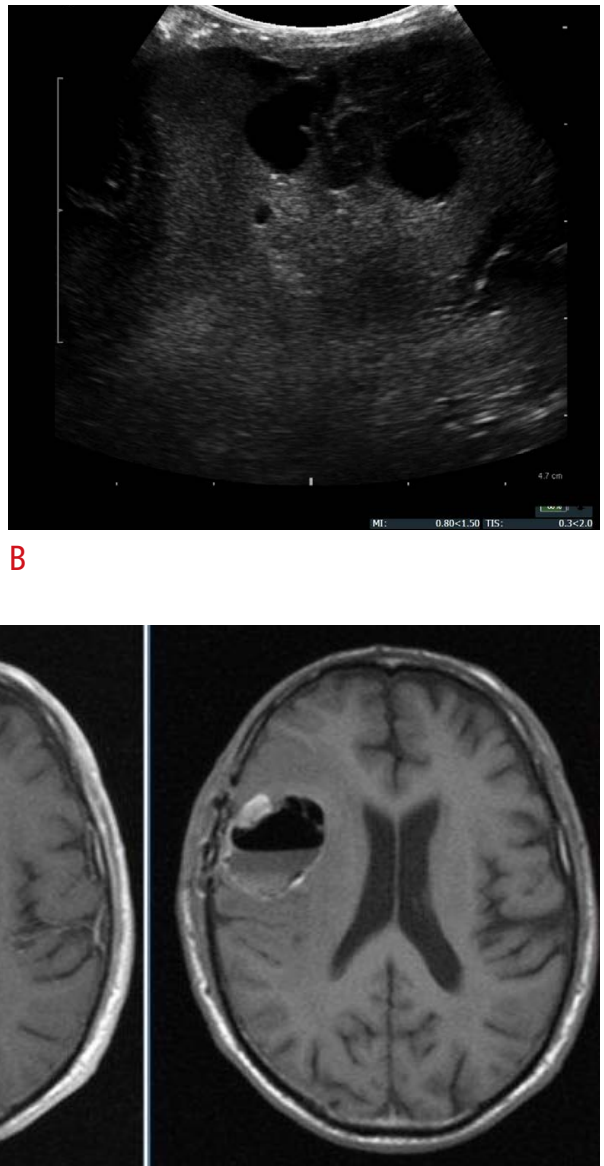

C

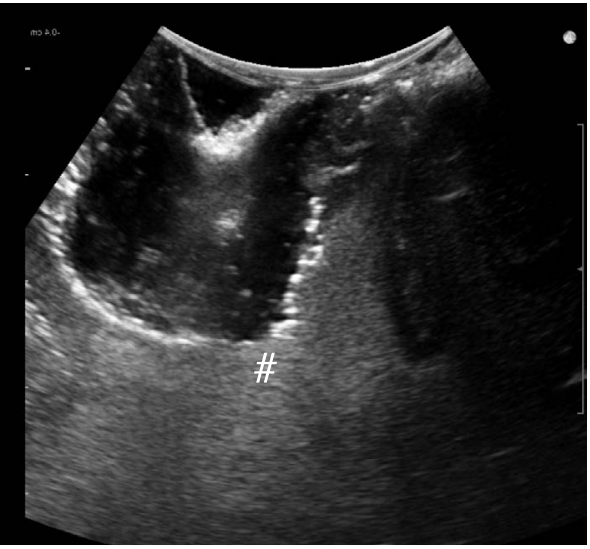

Tumor bulk

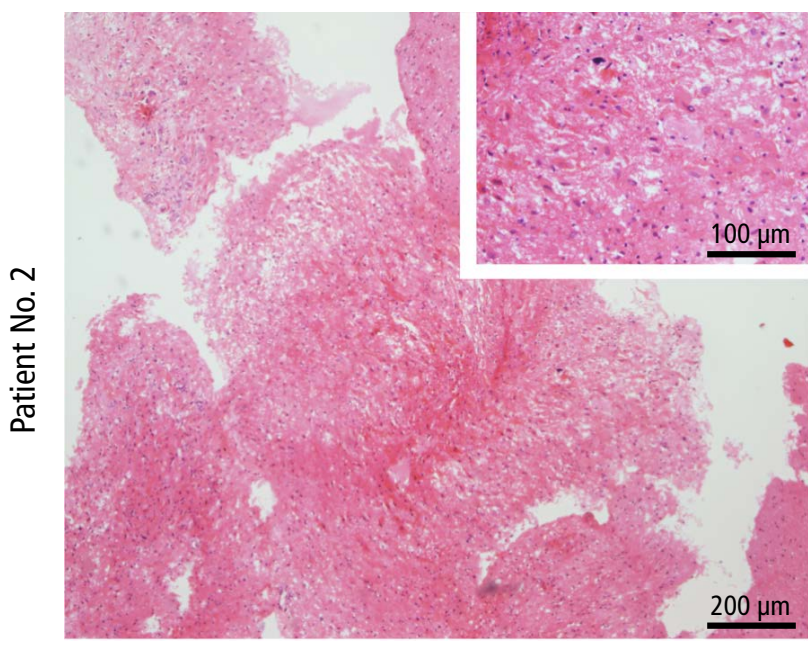

$\mathrm{E}$

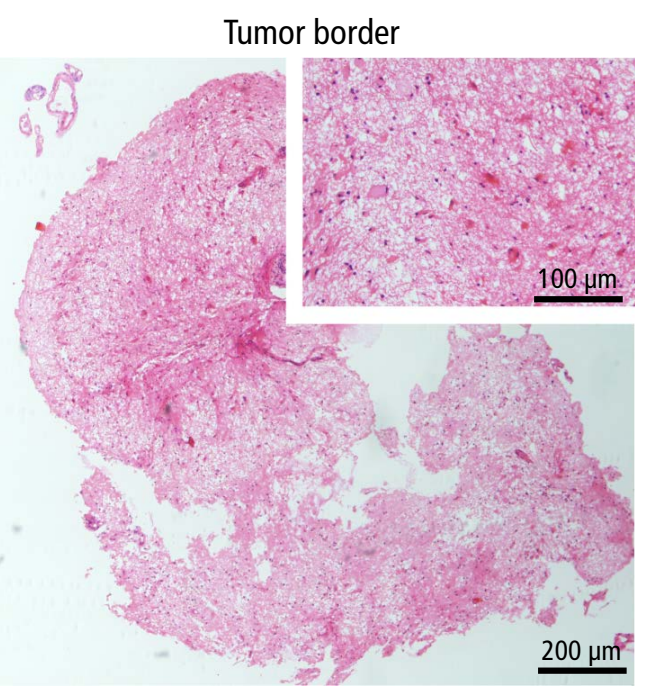

$\mathrm{F}$

Fig. 2. A 74-year-old man with a right frontal glioblastoma, who underwent reoperation 8 months after the initial operation.

A. Axial magnetic resonance imaging (MRI) (T1, contrast-enhanced) demonstrates the polycystic nonhomogenous tumor. B. This image could be used to navigate using intraoperative ultrasonography (IOUS) before resection (transverse image). C. Transverse IOUS reveals moderately hyperechoic tissue. In this localization, the sample 2 was obtained (\#). D. Axial postoperative MRI (left, contrast-enhanced; right native) excluded solid contrast enhancement. E, F. Histopathology using H\&E staining found that the hyperechoic tissue (tumor bulk) (E) contained tumor tissue, glial tumor cells, endothelial proliferation, and necrosis. Despite the moderately hyperechoic image on IOUS (C), the border was free of tumor cells, only reactive astrocytes are visualizable (F). 
due to an inability to distinguish between tumor and tumor-free tissue under the microscope, the signal was hypoechoic $(n=8)$ or moderately hyperechoic $(n=17)$. For moderately hyperechoic tissue, neuropathological examination revealed all possible histologic diagnoses: tumor, infiltration (Fig. 1), or tumor-free tissue (Fig. 2). However, hypoechoic tissue rarely (only 1 of the 8 specimens) harbored tumor tissue $(\mathrm{P}=0.150)$.

\section{Discussion}

IOUS has been used for navigation and resection control in intracranial surgery for decades [1]. Previously, doubts have been raised [10] that this method can quantify the extent of residual tumor, especially during glioma resection, but it nonetheless has become accepted as a valuable tool contributing to control of the resection [5]. Innovative techniques, such as three-dimensional imaging and high-frequency ultrasonography [11], have reportedly improved operative results [12]. This study focused on the issue of relapsed high-grade gliomas occurring in previously irradiated tissue. It has recently been demonstrated that the extent of resection has an impact on survival not only after the first operation but in this situation as well [13]. To our best knowledge, this problem has been mentioned concerning subgroups in other studies but not analyzed systematically. Chacko et al. [8] investigated the use of IOUS at tumor borders but excluded seven of the 35 irradiated patients due to irregular tumor margins. Hammoud et al. [3] examined 38 patients harboring gliomas, of whom 13 underwent radiation therapy. The intraoperative results were compared to the extent of resection measured by postoperative MRI. According to their results, the extent of resection was poorly defined by IOUS in irradiated gliomas.

The explanation for this phenomenon seems to be simple: ultrasound depicts differences in tissue echogenicity. Normal brain tissue has a relatively low echogenicity and thus, hyperechoic tumors can be easily seen. However, radiation itself may cause elevations in tissue echogenicity. Focusing only on irradiated patients and using histopathological controls, we emphasize that IOUS cannot perfectly predict the extent of resection. However, isoechoic tissue seldom contains tumor tissue. Ultrasound studies, even if the results are stored on video, remain subjective to a certain degree, as is the intraoperative impression of complete resection. We attempted to overcome this by choosing experienced investigators, but this limitation remains. To keep the analysis simple, only three grades of echogenicity were used. Our results lead to the question of whether IOUS is useful in these cases. However, the main result of our studythat the tumor core could be identified as hyperechoic tissue in all cases-underscores the usefulness of IOUS. A neurosurgeon who is facing the special situation of relapsed glioma may appreciate this option for navigating towards the bulk of the tumor. This may indeed be helpful when three-dimensional IOUS, navigated IOUS [14-16], or intraoperative MRI (which, according to some investigators $[4,6]$, may be superior to IOUS) are not available. Contrast-enhanced ultrasonography may improve the specificity, but has not been used in relapsed tumors [17]. The special value of IOUS, its real-time character, cannot be overemphasized. The intraoperative situation may change rapidly due to the collapse of cysts or the occurrence of hemorrhages $[9,18]$, and every possible tool for visualizing such changes should be utilized.

In conclusion, although the results regarding the echogenicity of the tumor boundary were not significant in this study, most likely due to the small number of subjects, our results suggest that a hypoechoic border, when achievable, is more likely to represent a tumor-free margin.

ORCID: Kay Mursch: http://orcid.org/0000-0001-9818-292X

\section{Conflict of Interest}

No potential conflict of interest relevant to this article was reported.

\section{References}

1. Dohrmann GJ, Rubin JM. History of intraoperative ultrasound in neurosurgery. Neurosurg Clin N Am 2001;12:155-166.

2. Cheon JE. Intraoperative neurosonography revisited: effective neuronavigation in pediatric neurosurgery. Ultrasonography 2015;34:79-87.

3. Hammoud MA, Ligon BL, elSouki R, Shi WM, Schomer DF, Sawaya R. Use of intraoperative ultrasound for localizing tumors and determining the extent of resection: a comparative study with magnetic resonance imaging. J Neurosurg 1996;84:737-741.

4. Tronnier VM, Bonsanto MM, Staubert A, Knauth M, Kunze S, Wirtz CR. Comparison of intraoperative MR imaging and 3D-navigated ultrasonography in the detection and resection control of lesions. Neurosurg Focus 2001;10:E3.

5. Woydt M, Krone A, Becker G, Schmidt K, Roggendorf W, Roosen K. Correlation of intra-operative ultrasound with histopathologic findings after tumour resection in supratentorial gliomas: a method to improve gross total tumour resection. Acta Neurochir (Wien) 1996;138:1391-1398.

6. Gerganov VM, Samii A, Akbarian A, Stieglitz L, Samii M, Fahlbusch R. Reliability of intraoperative high-resolution $2 \mathrm{D}$ ultrasound as an alternative to high-field strength MR imaging for tumor resection control: a prospective comparative study. J Neurosurg 2009;111:512-519.

7. Wang J, Liu X, Hou WH, Dong G, Wei Z, Zhou H, et al. The 
relationship between intra-operative ultrasonography and pathological grade in cerebral glioma. J Int Med Res 2008;36:14261434.

8. Chacko AG, Kumar NK, Chacko G, Athyal R, Rajshekhar V. Intraoperative ultrasound in determining the extent of resection of parenchymal brain tumours: a comparative study with computed tomography and histopathology. Acta Neurochir (Wien) 2003; 145:743-748.

9. Allouch H, Pfeifenbring S, Behnke-Mursch J, Halatsch ME, Mursch K. Real-time ultrasound monitoring during intracranial needle biopsies: operative results and detection of complications in 100 cases. World Neurosurg 2014;82:202-206.

10. Auer LM, van Velthoven V. Intraoperative ultrasound (US) imaging: comparison of pathomorphological findings in US and CT. Acta Neurochir (Wien) 1990;104:84-95.

11. Serra C, Stauffer A, Actor B, Burkhardt JK, Ulrich NH, Bernays RL, et al. Intraoperative high frequency ultrasound in intracerebral highgrade tumors. Ultraschall Med 2012;33:E306-E312.

12. Selbekk T, Jakola AS, Solheim O, Johansen TF, Lindseth F, Reinertsen I, et al. Ultrasound imaging in neurosurgery: approaches to minimize surgically induced image artefacts for improved resection control. Acta Neurochir (Wien) 2013;155:973-980.
13. Bloch O, Han SJ, Cha S, Sun MZ, Aghi MK, McDermott MW, et al. Impact of extent of resection for recurrent glioblastoma on overall survival: clinical article. J Neurosurg 2012;117:1032-1038.

14. Lindner D, Trantakis C, Renner C, Arnold S, Schmitgen A, Schneider J, et al. Application of intraoperative 3D ultrasound during navigated tumor resection. Minim Invasive Neurosurg 2006;49:197-202.

15. Unsgaard G, Ommedal S, Muller T, Gronningsaeter A, Nagelhus Hernes TA. Neuronavigation by intraoperative three-dimensional ultrasound: initial experience during brain tumor resection. Neurosurgery 2002;50:804-812.

16. Unsgaard G, Selbekk T, Brostrup Muller T, Ommedal S, Torp SH, Myhr G, et al. Ability of navigated 3D ultrasound to delineate gliomas and metastases: comparison of image interpretations with histopathology. Acta Neurochir (Wien) 2005;147:1259-1269.

17. Prada F, Bene MD, Fornaro R, Vetrano IG, Martegani A, Aiani L, et al. Identification of residual tumor with intraoperative contrastenhanced ultrasound during glioblastoma resection. Neurosurg Focus 2016;40:E7.

18. Allouch H, Behnke-Mursch J, Mursch K. Intra-operative diagnosis and image-guided management of an intracerebral haemorrhage occurring during ultrasound-guided biopsy. Acta Neurochir (Wien) 2007;149:91-93. 